\title{
Lüge und Realismus: Italo Calvinos La giornata di uno scrutatore und Louis Aragons Le mentir-vrai
}

Mensonge et réalisme: La giornata di uno scrutatore d'Italo Calvino et Le mentir-vrai de Louis Aragon

Lies and realism: Italo Calvino's La giornata di uno scrutatore and Louis Aragon's Le mentir-vrai

\section{Heinz Thoma}

\section{OpenEdition}

\section{Journals}

Édition électronique

URL : http://journals.openedition.org/ceg/1422

DOI : $10.4000 /$ ceg. 1422

ISSN : 2605-8359

\section{Éditeur}

Presses Universitaires de Provence

Édition imprimée

Date de publication : 15 juin 2015

Pagination : 63-75

ISBN : 978-2-85399-993-9

ISSN : 0751-4239

\section{Référence électronique}

Heinz Thoma, «Lüge und Realismus: Italo Calvinos La giornata di uno scrutatore und Louis Aragons Le mentir-vrai », Cahiers d'Études Germaniques [Online], 68 | 2015, Online erschienen am: 17 Dezember 2017, abgerufen am 05 Dezember 2020. URL : http://journals.openedition.org/ceg/1422 ; DOI : https://doi.org/10.4000/ceg. 1422 


\section{Lüge und Realismus: Italo Calvinos La giornata di uno scrutatore und Louis Aragons Le mentir-vrai}

Heinz THOMA

Martin-Luther-Universität, Halle-Wittenberg

\section{Einleitung}

Lüge und Realismus ist ein schwieriges und zugleich produktives Thema. Am einfachsten scheinen die Dinge noch zu liegen, wenn die Lüge als Thema verhandelt wird und obendrein der Rettung dient, so z. B. in Jakob der Lügner (1969) von Jurek Becker, wo der Protagonist durch die Erfindung, er besitze ein Radio und wisse so vom nahenden Vormarsch der Russen, d.h. der sowjetischen Armee, die Hoffnung der Ghettobewohner am Leben erhält. Zugleich produziert diese gut gemeinte Lüge aber Gefahren, erzeugt weitere Lügen, führt auch zu Selbstmord und Verfolgung. Schließlich belässt der Autor durch seine Erzählführung den Handlungsausgang im Blick auf das Schicksal des Protagonisten in einer quälenden Mehrdeutigkeit. Sicher ist nur die tatsächliche Ankunft der Roten Armee.

Jurek Beckers Text bewegt sich temporal in der Nähe der beginnenden Revisionen und Umdeutungen des zeitgenössischen Geschichtsbilds. Zu diesen gehört die Demontage des Résistance- bzw. Resistenza-Mythos, in der scheinbar feststehende Wahrheiten $\mathrm{zu}$ Widerstand und Antifaschismus, oft auch mit durchsichtigen antikommunistischen Intentionen, hinterfragt werden. Begünstigend für die Absicht der symbolischen Delegitimierung der Linken waren die Enthüllungen auf dem XX. Parteitag der KPDSU, welche auch die Glaubwürdigkeit bei der Suche nach einer politischen Alternative zum kapitalistischen System erschwerten. Die Krise der marxistischen Linken betrifft hierbei nicht nur ihr politisches Erscheinungsbild, sondern auch ihr ästhetisch-politisches Programm des sozialistischen Realismus. Im französischen Zusammenhang ist Roger Garaudys auch als Selbstkritik verfasster Text D’un réalisme sans rivages $(1963)^{1}$ hierfür ein sprechendes Dokument. Der führende Theoretiker des PCF, der später zum Islam übertreten wird, plädiert für eine künstlerisch-ästhetische programmatische Weite, die viele, darunterAragon, begrüßen, manche Kritiker indes Uferlosigkeit nennen. In Frankreich existiert im literarischen Feld als Konkurrent seit 1960 OULIPO, ein avantgardistischer Schriftstellerkreis für

Roger Garaudy, D’un réalisme sans rivages, Paris, Plon, 1963. 
potentielle Literatur (Ouvroir de littérature potentielle), der vor allem auf formale Experimente setzt. Ähnliches Interesse an einer formalen Erneuerung findet sich in der italienischen Neoavanguardia und dem Gruppo 63, dem neben Umberto Eco u.a. auch der marxistische Dichter Eduardo Sanguineti (1930-2010) angehört. In diese Zeit intensiver literarischer und kultureller Debatten fallen ebenso die im Folgenden zur Erörterung stehenden Erzählungen, Italo Calvinos (1923-1985) La giornata di uno scrutatore $(1963)^{2}$ und das den Titel eines Oxymorons ${ }^{3}$ tragende Fragment einer Autobiographie Le mentir-vrai (1964) ${ }^{4}$ von Louis Aragon (1897-1982). Beide haben auf je verschiedene Weise im Zentrum das Problem der Mimesis von Wirklichkeit in Verbindung mit dem Thema der Lüge bzw. des Lügens.

\section{Der Traditionsvorlauf}

Aus der Philosophie kennen wir das logische Problem, das der Kreter darstellt, der behauptet, alle Kreter seien Lügner. Derlei schützt davor, wahr und falsch allzu strikt trennen zu wollen und führt auf das Feld, wo sich beide begegnen, das der Fiktionalität. Platon hält im zweiten Buch der Politeia bekanntlich Hesiod und Homer als untauglich für die Erziehung der Wächter, da sie den Göttern unwahre Taten unterstellen und Zeus für des Guten wie auch des Schlimmen fähig halten, ihm gar trügerische Gestaltwechsel zutrauen. Diese Erfindungen der Dichter seien Lügen. Platon ist es aber auch, der befindet, dass die Seele in einem Zustande der wahren Lüge sein könne - für ihn ein abscheulicher Zustand - und dass, diskursiv gesehen, Lüge in gewissem Sinn höher einzustufen sei als die wahrheitstreue Rede. Denn der Lügner bedarf, will er zusammenhängend und widerspruchsfrei erzählen, um nicht entdeckt zu werden, eines besseren Gedächtnisses, stärkerer Vorstellungskraft und gewiss einer höheren Intelligenz und Phantasie als derjenige, der nur wahrheitsgemäß von sich gibt, was er erlebt hat. Noch Hanna Arendt wertet in Wahrheit und Politik (1963) den Lügner insofern auf, als sie ihn vom Verlogenen unterscheidet, der seine Lügen als solche nicht wahrzunehmen in der Lage ist. Jedenfalls, so Arendt, sei die Wahrheit nur beim Lügner noch zuhause.

Philosophisch gesehen ist der Lügner also ein gnoseologisch interessanter Fall und als Dichter eher gefährlich. In der Literatur hat die Lüge bzw. die Auseinandersetzung mit ihr ebenfalls eine weit zurückreichende Anciennität, nicht zuletzt wegen der Verteidigungsposition gegenüber der Philosophie. Am bekanntesten ist wohl der Eintrag in Horaz' Ars poetica, der von Homer sagt, er lüge

2 Italo CAlvino, La giornata di uno scrutatore, Turin, Einaudi, 1963, im Folgenden zitiert nach Milano, Mondadori, 1994; dt. Der Tag eines Wahlhelfers, in Italo CaLvino, Marcovaldo, Frankfurt a. M., S. Fischer, 1964, S. 139-206.

3 Auf der Ebene der politischen Höflichkeit findet diese rhetorische Figur ein paar Jahre später ihre Entsprechung in der Antwort Mitterands, mit der dieser 1972, nach der Verabschiedung des gemeinsamen Programms der Linken in der hochgestimmten Pariser Mutualité, Georges Marchais’ Bitte, ob man sich denn nun duzen könne, wie folgt beschied: „Si vous voulez“.

4 Louis Aragon, Le mentir-vrai, Paris, Gallimard, 1980, S. 7-47; dt. Das Wahr-Lügen, Berlin, Volk und Welt, 1980, S. 5-43. Nach diesen beiden Ausgaben wird zitiert. 
schön und mische wahr und falsch so voller Kunst ineinander, dass das Ganze wie aus einem Stücke scheine. ${ }^{5}$ Mimesis von Wirklichkeit ist, wo nicht eine Form der Lüge, so doch gleichsam mit ihr verschwistert, jedenfalls zumindest deren entfernte Verwandte. Dies gilt auch für die Wahrheit, wie der Begriff der Wahrscheinlichkeit in der ästhetischen Diskussion zeigt. Die Ambiguität geht in beide Richtungen. Terminologische Sicherheit sucht die Antike mit dem Begriff der fabula, sie ist im Unterschied zur historia keine wahre, und im Unterschied zum argumentum eine falsche, also erlogene bzw. erdichtete Erzählung. Im Christentum geht es elementarer zu. Nach Johannes 8, 44 ist der Teufel in Gestalt der Schlange der Vater der Lüge. Die auf die Fiktion ausgedehnte Haltung, die Literatur sei Teufels- und Blendwerk, lässt sich bis in die Polemiken gegen den Roman des 18. Jahrhunderts verfolgen.

Blickt man auf die Anfänge des bürgerlichen Realismus zurück, die in das Zeitalter der Aufklärung fallen, so ist dort der Antipode der höfische-galante Roman des 17. Jahrhunderts mit seinen unzähligen und unwahrscheinlichen Handlungsführungen wie mit seinen garantierten glücklichen Ausgängen. „C'est du roman" lautet im 18. Jahrhundert die Formel für ein unter dem Aspekt der Wahrheit und Wahrscheinlichkeit unhaltbares, erfundenes Geschehen. Im Deutschen haben wir hierfür die Wendung des Romanesken. Die Funktion des höfisch-galanten Romans war die Zerstreuung des Adels. Dagegen versucht man dann eine neue, wenn man so will, bürgerliche Erzählweise im Namen von Wahrheit und Moral. Diderot unterscheidet im poetologischen Nachtrag zur Novelle Les Deux Amis de Bourbonne (1772) drei Arten von Erzählungen: den conte merveilleux (Homer), den conte plaisant (La Fontaine) und den conte historique mit Cervantes und Marmontel. Dem conte historique, modern gesprochen, der realistischen Erzählung, gilt sein Augenmerk. Ihr Erzähler soll nach Diderot Lüge und Wahrheit gut verbinden, er muss „véridique et menteur" sein. Hierbei darf er kein platter und kalter Lügner (,menteur plat et froid“) sein, soll vielmehr interessieren, rühren, mitreißen, und wollen, dass man ihm glaubt. Wie also richtig täuschen? Der Erzähler muss seine Geschichte mit kleinen Umständen (,petites circonstances“) so durchsäen (,parsemer"), dass der Leser nicht umhin kann zu sagen: „Meiner Treu, das ist wahr“ („Ma foi, cela est vrai“ $).{ }^{6}$ Seine eigene Erzählung gestaltet Diderot mit vielfältigen Kunstmitteln so, dass sie letztlich nur eine Lesart erlaubt. „Die Sache sagen, wie sie ist“ („dire la chose comme elle est" ${ }^{\text {") }}$, heißt dann in dem gleichzeitig zur Erzählung unternommenen Roman Jacques le Fataliste et son maître aus dem Mund des Dieners die ideale, auch für die Fiktion geltende, Äußerungsweise.

Während Diderot seinen Realismus gleichsam materialistisch zu begründen sucht und die Raffinesse seiner erzählerischen Konstruktion exklusiv im Dienst der Aufklärung des Lesers steht, driftet die gemeine moralische Erzählung der Aufklärung durch meist auf Rührung zielende Konfliktlösungen oft in den Kitsch ab, eine der manifestesten literarischen Formen der Lüge, die, nicht zu vergessen, gleichwohl

,[...] atque ita mentitur (sc. der Dichter), sic veris falsa remiscet, primo ne medium, medio ne discrepet imum." Epistula ad Pisones [De arte poetica], in Q. Horatius Flaccus, Briefe, Berlin, Weidmannsche Verlagsbuchhandlung, 7. Aufl., 1961, V. 151-152.

6 Denis Diderot, Contes et romans, hrsg. v. Michel Delon, Paris, Gallimard (Bibliothèque de la Pléiade), 2004, S. 448 und 449. 
eine Sehnsucht nach Harmonie bedient. ${ }^{7}$ Die Prosafiktion des Höhenkamms der Literatur im 19. Jahrhundert behält die Frage nach der Wahrheit des Erzählten durch die Konkurrenz mit den Wissenschaften im Zentrum ihres Interesses. Balzac, so das Vorwort zur Comédie humaine, will der Linné der sozialen Klassen werden. Die Leser verbergen in ihrer Bibliothek ihren Sade hinter dem hl. Chrysostomus, frequentieren auch gern einen Autor der Scheinheiligkeit wie Octave Feuillet (1821-1890), mit dem sie ihre Selbstidealisierung pflegen. Und Lügen kommen selbstverständlich als Sachverhalt vor. ${ }^{8}$ Jedoch bleibt die Erzählhaltung am Kausalitätsmodell der Naturwissenschaften orientiert. Dies gilt nicht nur für Balzac, sondern auch für den kühl sezierenden Flaubert und den der Vererbungslehre folgenden Zola.

Da Aragon sich in Das Wahr-Lügen nicht nur mit der Mimesistheorie auseinandersetzt, sondern er dies zugleich am Beispiel eines Autobiographieversuchs unternimmt, bedarf es noch eines kurzen Blicks auf eine andere Erbschaft, jene Rousseaus. Nach der langen Reihe der vergeblichen theoretischen und narrativen Versuche Rousseaus, ein transparentes gesellschaftliches Zusammenleben schlüssig zu denken, situiert er sich zum Ende seines Schaffens auf dem anderen Pol, jenem des isolierten Individuums. Nur für dieses ist Transparenz möglich, heißt es in den zwischen 1765 und 1770 verfassten Confessions. In diesen finden wir an einer der Schlüsselstellen einen Sachverhalt vermuteter Lüge. Es handelt sich um die berühmte Kammepisode, in welcher Jean-Jacques als Jugendlicher einen von der Magd gereinigten Kämme seiner Ziehmutter, die zum Trocknen ausgelegt waren, in deren Abwesenheit zerbrochen haben soll: „Man konnte mir das geforderte Geständnis nicht entreißen." ${ }^{\text {"9 }}$ Und das erzählende Ich beschwört noch aus fast 50jähriger Distanz seine Unschuld. Im Nachgang zu dieser Szene verrät Rousseau, dass er gleichwohl gelegentlich ein kleiner Lügner war. Wichtiger als diese vielleicht unbewusste Selbstrelativierung ist aber, dass seine Darstellung von Anfang an denselben Anspruch auf Wahrheit erhebt wie Diderots objektivierendes Erzählkonzept. Personale Authentizität und „die Sache zu sagen, wie sie ist“, gehören beide zum Erbe der Aufklärung.

Mit der Wissenschaftsorientierung scheint für die Fiktion der Weg zur Selbstproblematisierung ihrer mimetischen Konstruktionen vorerst definitiv verschlossen. Mit der Krise des wissenschaftlich verbürgten Fortschrittskonzepts um 1900 und der tradierten Raum- und Zeitvorstellungen in der Physik ${ }^{10}$ beginnt sich indes das narrative Paradigma wieder der keineswegs definitiv beantworteten Frage von Wahr und Falsch zuzuwenden. Zeit und Raum werden nun dem Subjekt

\footnotetext{
Vgl. Kathrin ACKermann, Von der philosophisch-moralischen Erzählung zur modernen Novelle: ,contes ' und ,nouvelles' von 1760-1830, Frankfurt a. M., Klostermann, 2004.

8 Vgl. etwa die für das Thema der Desillusion im frühen 19. Jahrhundert typische Szene aus Alfred de Mussets Confessions d'un enfant du siècle (1836), wo man sich über dem Tisch liebevolle Blicke wirft und unter dem Tisch eine neue Beziehung anbahnt.

9 „On ne put m'arracher l'aveu qu'on exigeait.“ Jean-Jacques Rousseau, Les Confessions, Euvres complètes, hrsg. v. Bernard Gagnebin und Marcel Raymond, Paris, Gallimard (Bibliothèque de la Pléiade), 1959, S. 19.

10 Vgl. Vf., „Krise der Fortschrittsauffassung und Strukturreflexion auf die bürgerliche Formation“, in Georg Neugebauer et al. (Hrsg.), Aufklärung um 1900. Die klassische Moderne streitet um ihre Herkunftsgeschichte, München, Fink, 2015, S. 63-82.
} 
anheim gestellt, auch die Art der Darstellung selbst ist wieder stärker Problem und Gegenstand der Reflexion. Bergsons Philosophie, Prousts Recherche und Gides Les Faux-monnayeurs (1925) sind hierfür sprechende Beispiele. Einsteins Relativitätstheorie ist nur die Spitze sich verlierender Selbstverständlichkeiten. Hierhin gehört auch der Erfinder des Metatheaters Luigi Pirandello, bei dem schon in der Prosafiktion die Marquise nicht mehr zu einer bestimmten Uhrzeit aus dem Haus zu gehen hat. Valéry, Breton und Robbe-Grille nehmen seine zum Allgemeinplatz werdende Formel auf. Die veränderte Subjektstellung und die mit ihr einhergehenden Wahrnehmungsveränderungen thematisiert ausführlich Musils erster Band von Der Mann ohne Eigenschaften (1930). ${ }^{11}$ Um 1950 setzt der Nouveau Roman schließlich zielstrebig die bisher wahrheitsverbürgenden Koordinaten von Chronologie, Kausalismus, identitätssicheren Protagonisten etc. auch für die Er-Erzählung außer Kraft. Deswegen hatte Brecht zuvor schon durchaus Recht, wenn er im Streit mit Georg Lukács eine Wiederholung des auf agonalen Individuen basierenden Realismus des 19. Jahrhunderts aus der Perspektive des Proletariats, jedenfalls für die kapitalistische Sphäre des 20. Jahrhunderts, für einen ungeeigneten narrativen Zugang hielt. Zu dieser innerlinken Debatte gehört auf andere Weise auch diejenige, die in Italien zwischen dem populistischen Neorealismus und dem sozialistischen Realismus am Beispiel des Romans Metello (1955) von Vasco Pratolini sich entzündete.

\section{La giornata di un scrutatore}

Italo Calvinos rund achtzigseitiger Text hat zum Gegenstand die Durchführung der Parlamentswahl von 1953, genauer den Wahlablauf in einer großen, von der katholischen Kirche geleiteten und kontrollierten Behindertenanstalt, dem sogenannten Cottolengo in Turin. Die Hauptperson, ein Mitglied der Kommunistischen Partei mit liberalen Überzeugungen namens Amerigo Ormea ist Wahlhelfer. Der italienische Terminus scrutatore ist, wie der an den Entdecker Vespucci erinnernde Vorname des Protagonisten, auf die Haltung der Erkundung ausgerichtet, im Unterschied zum in der Übersetzung gewählten neutraleren und im Deutschen geläufigen Terminus. Die Er-Erzählung mit stark personaler, verhalten kommentierter und korrigierender Erzählführung gehört sachlich insofern zu unserem Thema, als impostura, so der Vorwurf des Betrugs seitens der Linken am Wahlverfahren, als Synonym auch menzogna, die Lüge, mit sich führt. Zu dieser Semantik gehört ebenso die für diese Wahl von 1953 verabschiedete legge truffa, auf deutsch „Schwindelgesetz“, das vorsah, dem Wahlsieger auch bei der knappsten Mehrheit 65\% der Sitze zuzugestehen. Narrativ gesehen erscheint die an der Oberfläche realistische Erzählung zunächst wie ein Rückfall gegenüber Calvinos so genanntem phantastischem Erzählen der Trilogie der Antenati aus den 1950er Jahren (die sich im Übrigen durchaus als kontinuierliche Auseinandersetzung mit der

11 Vgl. hierzu Vf., ,,Von der Entdeckung des Ichs zur, Amputation des Individuums'- Subjektposition und Subjektkonstruktion an literarischen Beispielen“, Sitzungsberichte der Sächsischen Akademie der Wissenschaften zu Leipzig - Philologisch-Historische Klasse, Bd. 140. H. 1, Leipzig, 2007. 
Weltdeutung durch die Kommunistische Partei Italiens lesen lässt). Jedoch bereits der erste Satz des Textes hält zur Vorsicht an: „Amerigo Ormea ging um halb sechs morgens aus dem Haus. "12 Der Texteingang nimmt den bereits von Pirandello als lügenhafte Illusion denunzierten Erzähleinsatz auf und verwendet auch das passato remoto, das wie das französische passé simple die Sicherheit der Weltbeherrschung durch den Erzähler als gegeben signalisiert. ${ }^{13}$ Die temporale Verschiebung um eine halbe Stunde und auf den frühen Morgen statt den späten Abend ist indes schon ein Hinweis auf eine bewusste Instrumentierung dieses Erzählbeginns und keineswegs Indiz für eine simple Restitution realistischer Verfahren. Das Problem der Wahrheit bzw. der Lüge erscheint also zuerst in der narrativen Gestalt, bevor es sich im Thema der Wahlfälschung sachlich konkretisiert. Calvino gelingt im Folgenden der Spagat zwischen einerseits einer eindrücklichen Kritik an den Wahlpraktiken und Tricks der zahlenmäßig dominierenden christdemokratischen Wahlhelfer, die das Wahlgesetz extensiv formalistisch auslegen, um die Gültigkeit der auf diese Weise abgegebenen Stimmen zu erzwingen und sich skrupellos der großen Zahl an Nonnen bedienen, die den Wasserköpfigen, Hand- und Armlosen, Dementen und Gelähmten etc. stellvertretend die Hand beim Ankreuzen führen, und andererseits einer tiefer gehenden Reflexion des Protagonisten auf die Rechte und den Gleichheitsanspruch dieser Benachteiligten, die in dieser gleichsam unsichtbaren Stadt des sogenannten Cottolengo ihr Leben fristen und ihr Auskommen finden. In dieser Welt verliert der Held angesichts der geballten Anschauung des „Elends der Natur“, wie es heißt, zwischen den Gipsmadonnen und Heiligenfiguren, angesichts derer ihm die zeitgenössische Dominanz der abstrakten Kunst erklärlich wird, zeitweilig die moralische und ästhetische Orientierung:

In der Cottolengo-Welt (in unserer Welt, die „Cottolengo“ werden könnte oder es schon ist) gelang es Amerigo nicht mehr, die von ihm als richtig erkannte [...] moralische oder ästhetische Linie zu verfolgen. [...] Für einen einzigen Tag seines Lebens gezwungen, sich über das Ausmaß des sogenannten Elends der Natur klar zu werden [...], fühlte er, wie sich zu seinen Füßen die abgründige Leere aller Dinge auftat. Ob dies der Zustand war, den man als ,religiöse Krise“ bezeichnet? ${ }^{14}$

Die Welt insgesamt scheint Amerigo (oder dem Erzähler?) im Begriff, dem Cottolengo zu ähneln. Zwar versucht der historische denkende Mensch (lo storicista) in ihm, aus dieser Krise wieder herauszufinden, indem er sich die Möglichkeit besserer und zukunftsträchtiger baulicher Anlagen für Behinderte in einer besseren Gesellschaft vor Augen stellt, er wird aber letztlich eine gewisse Relativierung seiner fortschrittsgläubigen marxistischen Überzeugungen jedoch nicht mehr völlig los. Hierauf weist vor allem das Schlussbild der Erzählung am Ende des

12 Calvino, Der Tag eines Wahlhelfers, ,S. 139. „Amerigo Ormea uscì di casa alle cinque e mezzo del mattino." La giornata di uno scrutatore, S. 3.

13 Vgl. hierzu Roland Barthes, Le Degré zéro de l'écriture, Paris, Éditions du Seuil, 1953.

14 Calvino, Der Tag eines Wahlhelfers, S. 171. „Nel mondo-Cottolengo (nel nostro mondo che potrebbe diventare o già essere „Cottolongo“) Amerigo non riusciva più a seguire la linea delle sue scelte morali $[. .$.$] o estetiche. [...] Costretto per un giorno della sua vita a tener conto di quanto è estesa quella$ che vien detta la miseria della natura [...] sentivi aprirsi sotto ai suoi piedi la vanità del tutto. Era questa, che chiamano una ,crisi religiosa'?"La giornata, S. 45. 
Wahltags, welches eine gelungene Utopie der Gegenwärtigkeit entwirft: Unter den letzten Wählern des Tages erscheint ein Arbeiter ohne Hände, der sich mit zwei zylindrischen Stümpfen geschickt und schnell eine Zigarette anzündet und der all seine Fertigkeiten, darunter auch das Wählen und Falten des Stimmzettels sich und den Schwestern verdankt, wie er sagt. Dieser Dialog vollzieht sich in der Abendröte, während zwerg- und riesenwüchsige, lachende Frauen gemeinsam einen Karren mit Reisig voran schieben, den Hof fegen und in einem auf zwei Fahrräder montierten Kessel ,vielleicht“", die Suppe transportieren: „Auch die allerletzte unter den Städten der Unvollkommenheit hat ihre Stunde der Vollkommenheit dachte der Wahlhelfer, hat die Stunde, den Augenblick, da eine jede Stadt die Stadt ist. "15

In die Darstellung des Wahltags verwoben ist die Beziehung Amerigos zu seiner Freundin, mit der er ein oberflächliches Verhältnis zu haben glaubt, die ihn am Telefon mit der Bemerkung ihrer Schwangerschaft überrascht und mit dem Hinweis, sie reise am nächsten Tag, wie am Vortag besprochen, tatsächlich zu ihrer Tante nach London (sc. vermutlich, um abzutreiben), wozu er sie ja ermutigt habe. Aus seiner Sicht hat sie ihn zu wörtlich verstanden, wie immer, wenn er etwas sage. Das Paar hat offensichtlich verschiedene Wege des Verstehens und der Mitteilung, da sie sich wenig gleichen, ohne sich indes zu belügen. Und doch findet der Protagonist im Verlauf der Telefonate des Tages, sichtlich befördert durch seine Anschauung des Cottolengo, zur Akzeptanz der prälogischen Art der Partnerin, wie er deren Charakter nennt, und ihres momentgebundenen Verhaltens, das, so kann man ex post schließen, ein Äquivalent in jenem, nur für die Dauer eines Augenblick möglichen Zustand einer perfekten Stadt zu haben scheint. Calvino, der, wie auch im Übrigen der frühe Aragon, einen patriarchalisch gefärbten Kult des Weiblichen pflegt, ist in seiner Erzählung von 1963 bereits auf dem Weg zu einer neuen Phase seiner von Beginn an anthropologisch basierten Anschauung der Welt. Die ,Schwindel'-Wahl im Cottolengo ist Anlass zu einem hochreflexiven Realismus zweiter Ordnung, mit dem die Forschung bisher nicht zu recht kam. Man hat indes zeigen können, dass dieser Realismus an die im Cavaliere inesistente (1959) ungelöst gebliebenen Fragen der Moral anschließt und er zugleich die Schriftstellerexistenz des 20. Jahrhunderts im Spannungsfeld von Arbeiterklasse und allgemeiner gesellschaftlicher Verantwortung vorführt. In dieser Hinsicht, wie in den unauffällig und doch sichtbar mitgeführten gnoseologischen und sozialen bzw. politischen Referenzebenen, bildet La giornata di uno scrutatore gleichsam eine Summe des bisherigen Werks des Autors. ${ }^{16}$

15 Der Tag eines Wahlhelfers, S. 207. „Anche l'ultima città dell'imperfeziona ha la sua ora perfetta, pensò lo scrutatore, l'ora, l'attimo, in cui ogni città c'è la citta.“ La giornata, S. 83.

16 Siehe die glänzende Interpretation des Textes durch Kristin ReICHEL, L'uomo completo, Anthropologie und Gesellschaft in Poetik und Praxis von Italo Calvino, Würzburg, Könighausen \& Neumann, 2006, S. 329-423. Sie liest La Giornata im Prisma von Barthes, Hegel, Diderot, Popper, L. Goldmann und Italo Balbo als mythen- und ideologiekritischen Text und erkennt im Cottolengo auch Dantes Divina commedia wieder. 


\section{Le mentir-vrai}

Auch Aragons autobiographische Erzählung steht im literaturhistorisch präzise benennbaren Kontext des Nouveau Roman wie der linken Debatten um ein epochengerechtes Schreiben. ${ }^{17}$ Zugleich fallen in die Jahre der Entstehung von Aragons Text zahlreiche autobiographische Versuche von Sartre, Simone de Beauvoir u.a. Vorauszusetzen ist, dass Aragon nach seinen surrealistischen Schreibanfängen ab 1930 zum sozialistischen Realismus wechselte und in dieser, von ihm in keiner Hinsicht schlicht geführten Erzählhaltung einen Romanzyklus Le Monde réel nach der Art des 19. Jahrhunderts schrieb, der die soziale und politische Totalität Frankreichs des frühen 20. Jahrhunderts abbilden sollte. ${ }^{18}$ Um 1964 begann er mit der Umschreibung des Zyklus. In diese Phase gehört die Revision der Cloches de Bâle. Dieser erste Band des Zyklus handelt von der Belle Époque und führt das ganze politische Personal der dritten Republik vor, teilweise auch Personen, die zu unserer Erzählung gehören. Die Umschreibung des Romans begründete Aragon mit der veränderten Sichtweise seiner Zeit. Der Titel von Mentir-vrai ist zugleich die Formel seiner damaligen Poetik des Erzählens, wie er auch anderwärts betont. ${ }^{19}$

Im Gehalt bildet die Erzählung einen Ausschnitt einer echten und zugleich fiktiven Biographie: das Jahr der Erstkommunion, eines Jungen, dessen narrative Situierung und Fakten auf den elfjährigen Louis Aragon (1897-1982) verweisen. Die Handlung spielt also im Jahr 1908. Die Darlegung aus der Sicht eines Kindes ist funktional ähnlich jener aus der Sicht des Edlen Wilden, topisch für Situationen der Verfremdung und der Dekuvrierung, die Lügen gut sichtbar machen kann.

Das erzählte jugendliche Ich hat keinen sicheren Namen, nennt sich Pierre oder Jacques oder Louis Aragon. ${ }^{20}$ Pierre nennt ihn auch das erinnernde Ich. Der junge Pierre ist ein Bastard. Der Vater ist hoher Beamter und Politiker im republikanischen Geschäft. Er ist Laizist, und nahm bereits an den antiklerikalen Maßnahmen unter Louis Grévy (1817-1891) vor der Jahrhundertwende aktiven Anteil. Zur erzählten Zeit ist er bereits 68 Jahre alt. Die Mutter undeutlichen Alters muss der Junge offiziell Marthe nennen, er gilt als Adoptivkind, dessen Vater und Mutter verstorben seien. Es handelt sich also um eine Kindheit im Horizont der Lüge. Wir sind in Paris. Der gelegentlich vorbeischauende Vater dringt auf Fechtunterricht, schenkt dem Sohn einen ausgedienten Zirkelkasten seiner legitimen Söhne und deren abgelegte deutsch-französischen und französisch-deutschen Wörterbücher. Sie enthalten um die Worteinträge gruppierte obszöne Kritzeleien. Der Junge verbirgt

\footnotetext{
17 Sie erschien zum ersten Mal im vierten Band der Euvres romanesques croisées d'Elsa Triolet et d'Aragon, Paris, Laffont, 1964, S. 263-297.

18 Les Cloches de Bâle 1934, Les Beaux Quartiers 1936, Les Voyageurs de l'Impériale 1942, Aurélien 1944, Les Communistes 1949-1951.

19 „[...] Le Mentir-Vrai est, plus qu'une nouvelle, un 'art romanesque"“ in CEuvres romanesques complètes, Paris, Gallimard, Bd. 1, 1997, S. 237, zit. nach Wolfgang BaBILAS, Études sur Louis Aragon, Münster, Nodus Publikationen, 2002, 2 Bde., Bd. 2., S. 879.

20 Tatsächlich ist Aragon uneheliches Kind von Louis Andrieux, einem Polizeipräfekten und nachmaligen Deputierten, der sozial aus der (protestantischen) Großbourgeoisie stammt, ideologisch dem Milieu der Freimaurer angehört. Die Mutter ist Marguerite Toucas-Massillon, sie kommt aus der katholischen Mittelbourgeoisie und führte eine Familienpension.
} 
diese Wörterbücher so gut wie möglich. Zum Kreis der engeren Familie, die sich mütterlicherseits vom Hofprediger Ludwigs XIV., Massillon, herleitet, gehört der Onkel Edouard, zeitweilig Sekretär des Vaters, mit eigenen politischen Ambitionen, auch ein zeitweiliger Zeitungsgründer im Dienst des Quai d'Orsay. Er ist ein Mann vieler Amouren und seichter Lektüren. Ferner ist da die Großmutter, eine dumme Gans, so der Erzähler, die zur Religion tendiert. Sie bekommt eine Tabaklizenz in Toulon zugeschoben und kontrolliert den Jungen. Schließlich ist da noch Paulette, eine Verwandte, die auf Kosten der Mutter lebt, später durch die Heirat mit einem General in die oberen Kreise gelangt und die Mutter zu bestimmten gehobenen Anlässen nicht einlädt. Kurz, der Junge gehört und doch auch nicht zur guten Gesellschaft. Die zwei familles d'esprit, die im Horizont Pierres erscheinen, sind der Katholizismus und der Republikanismus. Zeitlich liegt die Trennung von Staat und Kirche drei Jahre zurück.

Die weltanschaulichen Strömungen spiegeln sich auch in Pierres Freundschaften. Paul, der Lieblingsfreund, absolviert den Mathematikzweig und liebt populäre Bildergeschichten (LePionnier Cambembert). Er istPierres Freund fürWissenschaften und Reisepläne. Beide wollen sie Jules Vernes Programm weiterschreiben, planen eine Polarexpedition. Im fortgeschrittenen Planungsstadium wollen sie auch eine Frau mitnehmen. Guy, der zweite Freund, ist katholisch, Camelot du roi und Anhänger des Herzogs von Orleans. Pierre, der den sprachlichen Zweig besucht, ist ihm zugeneigt, weil er für seinen Hang zum Dichten Verständnis hat und er seine Verse schätzt, die er Paul nicht zeigt. Paul und Guy distinguieren sich gegenüber Pierre durch ihre teuren Bücher bzw. Schultaschen. Der Kreuzungspunkt dieser Freundschaft ist ein privates, geschlechtergemischtes katholisches Gymnasium, in dem der Bestschüler Pierre unter den Abbés Freunde gewinnt. Einer dieser Abbés nennt ihn Jacques. Als sich unter dem Eindruck der bevorstehenden Erstkommunion Pierres religiöse Leidenschaft steigert, sieht man ihn für das Priestertum vor. Die Religion ist indes für ihn eher eine Antihaltung, mit der er gegen den Vater reagiert und mittels derer er, durch seine Gebete, die Mutter zu schützen sucht. Kleine mystische Anwandlungen weisen auch in die erotische Richtung. Die Erstkommunion schließlich ist für ihn emotional eine herbe Enttäuschung. Am Ende der Erzählung sind jedenfalls die religiösen Anwandlungen verflogen, und es heißt, sich in der zeitlichen Gegenwart einzurichten: „Jedenfalls glaube ich an nichts mehr. Weder an Gott noch an den Nordpol und das Leben wird so sein wie es sein wird. "21 Dieses Fazit hat seinen Platz nach dem Hinweis auf eine aussichtslose Ferienliebe zu einer Vierzehnjährigen.

Die Freundinnen des Erzählers sind altersmäßig für ihn unerreichbar. Da ist die wesentlich ältere Catherine, die ihm Bücher leiht, so den für die Poetik bedeutsamen Roman Tolstois Anna Karenina. Wichtiger für Pierre ist aber Sonja, eine Mitschülerin russischer Herkunft, die sich wie die Figur eines Trivialromans als Zigeunerin verkleidet. Er hält sie für ein morganatisches Kind des Zaren und wähnt sich angesichts der Gemeinsamkeit in der Illegitimität der Abstammung schon in einer Sonderbeziehung mit der Angebeteten. In Wirklichkeit war die Großmutter

21 Aragon, Das Wahr-Lügen, S. 43. „De toute façon, je ne crois plus à rien. Ni à Dieu, ni au pôle Nord, et la vie sera ce qu'elle sera." Le mentir-vrai, S. 47. 
das illegitime Kind. Am Ende dieser Wirrungen greift er zu Goethes Werther und beklagt sich, er habe nicht einmal einen Wilhelm, dem er schreiben könne.

Goethes Sturm und Drang-Text gehört zu seinen zahlreichen Lektüren. Pierre liest, was ihm in die Hände kommt, Catherine ihm mitbringt und was er sich aus den Familienbibliotheken zusammenklaubt, etwa Télémaque, Chateaubriand, Marmontel, Le Jeudi de la jeunesse, Hector Malot (1830-1907). Einerseits handelt es sich um Autoren zweiter Ordnung, wie Malot, die im gewissen Sinn das Milieu, in dem er lebt, aus der Erwachsenenperspektive reproduzieren und ihn so über manches aufklären, dessen er noch nicht ansichtig geworden ist. Zum anderen sind dies aber auch Texte, die nur für einen frühreifen Jugendlichen taugen, und das ist er dann doch noch nicht ganz. So liest er parallel zu Rousseaus Confessions, die er nicht richtig versteht, die Confession de Nicaise, von Pierre Valdagne (1854-1937), ein Gesellschaftsautor zweiter Ordnung, der die Erinnerungen eines von seinen Schwestern verkauften jungen Mädchens feilbietet. Geständnis ist der Parameter für ein neugieriges Kind, das noch nicht viel weiß und nicht sortieren kann. Noch zu früh kommen für ihn auch die ersten Lieferungen von Jean-Christophe, ein Roman zur deutsch-französischen Verständigung (1904-1912) von Romain Rolland (1866-1944).

Der Unsicherheit der Identität des Heranwachsenden im Prisma der Erinnerung entsprechen Auslassungen zur Poetik seitens des erinnernden Ich.

Armer Junge im Spiegel. Du siehst mir nicht mehr ähnlich, und dennoch siehst du mir ähnlich. Ich bin es, der spricht. Du hast nicht mehr deine Kinderstimme. Du bist nur mehr eine Manneserinnerung, später. Wenn das hier dein Tagebuch wäre, stünde da der Preis deines Kreisels drin, das Thema des französischen Aufsatzes, die Besuche im Louis XVI.-Salon und die kleine Zwergdominoschachtel, die du gestern abend aus Vernis-Martins Vitrine stibitzt hast. Ich wiederhole mich fünfundfünzig Jahre später. Das entstellt die Wörter. Und wenn ich glaube mich zu betrachten, imaginiere ich mich. Das ist stärker als ich. Ich bringe Ordnung in mich hinein. [...] Ich glaube mich zu erinnern, aber ich erfinde mich. [...] Übrigens hieß ich nicht Pierre, lediglich Abbé Pangaud (und nicht Prangaud) nannte mich Pierre, er nannte mich nicht Jacques. [...] Diesen Guy pflückst du obendrein von einem merkwürdigen Baum. Wer weiß, wie er sich jetzt sieht, seine Pelerine... Und jedenfalls dachtest du (ich meine ,ich“), dachtest du (ich) nicht „Mama“ mit elf Jahren. [...] Im Augenblick legst du die Dinge übereinander. Ich...nun, der Grundstein ist gelegt, wir posieren. ${ }^{22}$

22 Das Wahr-Lügen, S. 7f. „Pauvre gosse dans le miroir. Tu ne me ressembles plus, pourtant tu me ressembles. C'est moi qui parle. Tu n'as plus ta voix d'enfant. Tu n'es plus qu'un souvenir d'homme, plus tard. Si c'était ton journal, il y aurait le prix de ta toupie, le sujet de la composition française, les visites dans le salon Louis XVI et la petite boîte de dominos nains que tu as chipée hier soir dans la vitrine de Vernis-Martin. Cinquante ans plus tard. Ça déforme les mots. Et quand je crois me regarder, je m'imagine. C'est plus fort que moi, je m'ordonne. Je rapproche des faits qui furent, mais séparés. Je crois me souvenir, je m'invente. [...] D'ailleurs, je ne m'appelais pas Pierre, c'était l'abbé Pangaud (et non Prangaud) qui m'appelait Pierre, et pas Jacques [...]. Ce Guy-là, aussi tu le cueilles dans un drôle d'arbre. Qui sait comme lui se voit maintenant, sa pèlerine... Et de toute façon, toi, je veux dire moi, tu je ne pensais pas Maman, à onze ans. Cela est un mensonge concerté, de faire croire que tu jouais ainsi double jeu entre toi et les autres. Cela viendra plus tard. Pour l'instant tu superposes. Je... enfin, c'est posé, nous posons." Le mentir-vrai, S. 9f. 
In dieser ersten größeren poetologischen Erörterung des sich erinnernden Ich lässt dieses sich über seine fiktiven Verfahren, etwas später auch über seine Mogeleien aus, spricht von der Zurechtrückung der Erinnerung, die er kindgerecht korrigiere. Es beginnt also mit der Beschreibung des so genannten Wahr-Lügens, dessen Erinnerungsstückchen, wie es anderwärts heißt, keine Photographie, sondern einen Karneval ergeben. Die Unzuverlässigkeit wird meist dem Jungen zugeschoben, gleichwohl ist sie immer die nachvollziehbare Konstruktion des erinnernden Ich, wobei die Parameter von Wahrheit und Wahrscheinlichkeit keineswegs aufgegeben werden. ${ }^{23}$ Das bleibt im Grundsatz in der Tradition Rousseaus, jedoch wird hier der Schwierigkeit authentischen Darstellens mehr Raum gegeben. In der zweiten größeren poetologischen Erörterung reflektiert der erinnernde Erzähler auf die Schreibzwänge, unter denen er selbst steht:

Es ist notwendig, dass meine Gestalt (Pierre) mit elf Jahren Rousseau und Valdagne gelesen hat. Wenn das ich wäre...Mir natürlich würden Sie alles durchgehen lassen. Ich bin eine ungewöhnliche Gestalt, nicht jeder hat das „Pariser Landleben“ (i.e. Le Paysan de Paris, H.T.) geschrieben, nicht jeder hat im Telemach (i.e. Télémaque von Fénelon, H.T.) lesen gelernt. Es ist notwendig, dass Pierre mit 11 Jahren Valdagne und Rousseau gelesen hat und vieles andere mehr [...], damit sich diese Sprache erklärt, die ich fünfundfünzig Jahr später spreche [...] wenn ich 1908 erstehen lassen will. [...] niemals würde jedenfalls dieser Junge dieses synkopierte Sprechen schreiben, dieses mündliche Französisch, das meiner Generation eigen ist. Ich kann ihm indes nicht, unter dem Vorwand, wahr zu lügen, das imaginäre Schreiben zudiktieren, das er hätte haben können, nur damit das zu den typischen Texten kleiner Jungen passt. Die Worte dieses Schülers, der mir ähnlich ist, dürften eben gerade nicht den meinen von damals ähneln, oder es wäre aus mit der Wahrscheinlichkeit. ${ }^{24}$

Ziel ist also, im Namen der Wahrscheinlichkeit wiederzugeben, wie Pierre das schriebe, was der Erzähler heute über ihn damals denkt. ${ }^{25}$ Etwas befremdlich wirkt angesichts solcher Subtilitäten jene andere Stelle, die mit Hinweisen auf Zensur und Selbstzensur und Tabus des 20. Jahrhunderts zu der Voraussage kommt, die „Realisten der Zukunft“ würden „,immer mehr lügen müssen, um die Wahrheit zu sagen." ${ }^{\text {26 }}$ Das ist angesichts der kommenden gesellschaftlichen und literarischen

23 Eine filigrane Auseinanderlegung des Lügenkontexts gibt Wolfgang BaBILAS, „Vérité' et 'mensonge' dans la nouvelle Le mentir-vrai de Louis Aragon“"in Études sur Louis Aragon, Bd. 2, S. 875-900.

24 Das Wahr-Lügen, S. 10f. „Il faut que mon personnage (Pierre) ait lu Rousseau et Valdagne à onze ans. Si c'était moi... Naturellement à moi, vous me passeriez tout. Je suis un personnage hors série, tout le monde n'a pas écrit Le Paysan de Paris, tout le monde n'a pas appris à lire dans Télémaque. Il faut que Pierre ait lu à onze ans Valdagne et Rousseau, et bien d'autres choses [...] pour expliquer ce langage que je parle cinquante-cinq ans plus tard [...] jamais de toute façon, cet enfant il n'écrirait ce parler syncopé, ce français oral, qui est de ma génération. Je ne puis pourtant pas, sous prétexte de mentir vrai, lui donner l'imaginaire écriture qu'il aurait $p u$ avoir pour que ça ne jure pas avec les textes des petits garçons typiques. Cet écolier qui me ressemble, il ne faudrait pas justement pas que ses mots ressemblent aux miens d'alors, ou adieu la vraisemblance." Le mentir-vrai, S. 12f.

25 Vgl. auch die abgründige Passage, in der der Erzähler bedauert, die Frische der Religiosität des Knaben nicht nachempfinden zu können und so ihm die Fähigkeit zu einem grundsätzlichen Neuanfang abgehe (Das Wahr-Lügen,S. 28).

26 Das Wahr-Lügen, S. 22 ; „Les réalistes de l'avenir devront de plus en plus mentir pour dire vrai.“ Le mentir-vrai, S. 24 
Entwicklungen von geringer prognostischer Kraft. Von romantheoretischem Interesse ist schließlich die Integration von Catherine Simonidzé, einer georgischen Anarchistin und Figur aus dem aus „Träumereien gemachte[n]“ ${ }^{27}$ Zyklus des Monde réel von 1934-1951, in Le mentir-vrai. Sie heiße in Wirklichkeit Elisabeth, so der ältere Erzähler, eine Figur also aus seinem Leben, ein Geschenk an den jungen Pierre, der es nicht annahm, sondern sich in Sonja verliebte. Diese, tendenziell die Mimesiskonzeption auflösende, jedenfalls verzweigende, Episode betrifft vor allem den älteren Erzähler.

\section{Fazit}

Mit beiden Autoren und ihren Erzählungen verlässt man die Enge mancher Erörterungen der 1950er Jahre. Beide Autoren zeigen, dass sie sich auf der Höhe der zeitgenössischen Debatten über den Roman befinden, hierbei jedoch eigenständige Positionen beziehen. Aragon, Jahrgang 1897, ist vielschichtiger und abgründiger als Calvino, näher auch an der bürgerlichen Schicht älterer Provenienz und raffinierter in der Evozierung von Täuschungen und Selbsttäuschungen, die gleichsam eine Wahrheit eigener Art hervorbringen. Hierzu eignet sich natürlich besonders auch der autobiographische Versuch, der einen Anspruch aufAuthentizität in der Art Rousseaus ausschließt, ohne indes auf den Zwang zur Plausibilisierung gänzlich zu verzichten. Dieser Tendenz zur bleibenden Weltanbindung fiktiven Geschehens, die sich auch in der Persistenz der ästhetischen Kategorie der Wahrscheinlichkeit manifestiert, dient nicht zuletzt der Ausgriff auf die Genese von Tolstois Roman Anna Karenina, mittels dessen Aragon das Romanschaffen und dessen Entstehungsbedingungen in den Horizont der Geschichte und damit unter das Signum der Veränderlichkeit stellt. Diese Sichtweise verändert auch die Antworten auf das Problem von Fiktion, Wahrheit und Lüge.

Im Vergleich zu Aragons Schreibweise ist die Erzählung des in einem naturwissenschaftlich geprägten Elternhaus aufgewachsenen und eine Generation jüngeren Calvino, Jahrgang 1923, ruhiger und elementarer. Die mise en abyme eines strikten Gegensatzes von Wahrheit und Lüge mit Mitteln der ironischen Distanz ist bei ihm weniger ausgeklügelt und ergibt sich weit stärker aus der Einsicht in elementare soziale und anthropologische Gegebenheiten. Zugleich hat die Historisierung der Lüge bei Calvino, trotz der Enge des gewählten Weltausschnitts, prospektiv die größere Chance zu einer mental und skriptural adäquaten Amplitude im Prozess der Wahrheitsfindung. Die anthropologische Suche nach dem „kompletten Menschen“ („uomo completo“) geht bei ihm offenere Wege, der Horizont wird die Welt, der Autor in Paris, den USA und in Rom zum Weltbürger und die gewählten literarischen Formen werden, auch durch die Mitgliedschaft in OULIPO, mit Le città invisibili (1972) und Palomar (1983) freier verfügbar, ohne dass sie den Wirklichkeitskontakt aufgäben. Die jeweiligen Unterschiede erklären sich vermutlich aus der Dichte und Bindungskraft des nationalen kulturellen Felds, das Calvino zum Zeitpunkt von

27 Das Wahr-Lügen, S. 36. 
La giornata di uno scrutatore zu verlassen im Begriff steht und aus dem Aragon sich nie zu entfernen gedachte. Dieser Gesichtspunkt bedürfte einer gesonderten Erörterung. Spannt man den ästhetisch-kulturellen Bogen zeitlich etwas weiter, so liegen sowohl Calvinos wie Aragons Text noch deutlich diesseits der Postmoderne, in deren kognitiv-pragmatischen wie ästhetischen Grundmustern sich die Wahrheitsfrage grundsätzlich zu verflüssigen beginnt - und damit das Thema der Lüge, jedenfalls im literarischen Feld, fürs erste gegenstandslos zu werden scheint. 
